九州大学学術情報リポジトリ

Kyushu University Institutional Repository

\title{
REVERSIBILITY OF CA-150 WITH SYMMETRY LOCAL STRUCTURE
}

INOKUCHI, Shuichi

Department of System Management, Fukuoka Institute of Technology

ISHIDA, Toshikazu

Faculty of Science and Engineering, Kyushu Sangyo University

https://doi.org/10.5109/4706230

出版情報: Bulletin of informatics and cybernetics. 53 (5)，pp.1-7，2021. 統計科学研究会 バージョン：

権利関係 : 


\section{REVERSIBILITY OF CA-150 WITH SYMMETRY LOCAL STRUCTURE}

by

Shuichi INoкUCHI and Toshikazu IshidA

Reprinted from the Bulletin of Informatics and Cybernetics Research Association of Statistical Sciences, Vol.53, No. 5

FUKUOKA, JAPAN

2021 


\title{
REVERSIBILITY OF CA-150 WITH SYMMETRY LOCAL STRUCTURE
}

\author{
By \\ Shuichi INoK UCHI ${ }^{*}$ and Toshikazu IshIDA ${ }^{\dagger}$
}

\begin{abstract}
The local structure of a cellular automaton is a set of its neighborhood that represent interacting cells and its local function that represent its interaction. In this paper we discuss the behavior, especially the reversibility of of CA-150 extended the local structure (neighborhood) symmetrically and show a relationship of the cell size and the neighborhood radius for CA-150 to be reversible.
\end{abstract}

Key Words and Phrases: Cellular automata, Reversibility, Local structure

\section{Introduction}

Since Feynman (1982) proposed the concept of quantum computing, various models of quantum computing have been proposed and studied. Watrous (1995) proposed a quantum cellular automaton as one of quantum computing models. This quantum cellular automaton with an infinite cell array has been shown to have the computational universality. On the other hand, the concept of quantum cellular automata with a finite cell array was also proposed and Inokuchi and Mizoguchi (2005) showed sufficient conditions for classical (discrete) cellular automata to be extensible to quantum cellular automata. One of the sufficient conditions is that classical cellular automata are reversible. The quantum cellular automata targeted by Inokuchi et al. (2008) and Inui et al. (2005) are extensions of the well-known 1-dimensional 2-states 3-neighborhood cellular automata that are reversible, and Schumacher and Werner (2004) consisted of a special cellular automaton whose reversibility is trivial.

Researchs on the reversibility of cellular automata and reversible cellular automata have been studied so far. Wolfram (2002) discussed the reversibility of various cellular automata with an infinite cell array, and showed that there are only six kinds of obvious reversible 1-dimensional 2-states 3-neighborhood cellular automata. Inokuchi, Honda et al. (2005) also reported that there exist non-trivial 1-dimensional 2-states 3 -neighborhood cellular automata with a finite cell array which are reversible.

Bhattacharjee and Das (2016) investigated the reversibility of 1-dimensional 3-neighborhood cellular automata with multi states of cells, and Bhattacharjee and Das (2019) introduced the semi-reversibility and classified cellular automata into three types with respect to the reversibility.

Morita and Harao (1989) and Morita (1995) showed the computational universality of

\footnotetext{
* Department of System Management, Fukuoka Institute of Technology, 3-30-1 Wajiro-higashi HigashiKu Fukuoka 811-0295 Japan. inokuchi@fit.ac.jp

$\dagger$ Faculty of Science and Engineering, Kyushu Sangyo University, 2-3-1 Matsukadai Higashi-Ku Fukuoka 813-8503 Japan.
} 
reversible cellular automata and proposed a method for constructing a reversible cellular automaton that simulate an irreversible cellular automaton. Nishio (2006) and Nishio (2007) investigated how the global behavior of cellular automata changes by changing not only their local functions but also their neighborhood, and proves that the reversibility and the neighborhood are independent for six 1-dimensional 2-state 3neighborhood cellular automata whose reversibilities are shown by Wolfram (2002). It is also proved that the surjectivity (injectivity) does not depend on the neighborhood in the linear cellular automata on a cell array where the Garden of Eden theorem holds.

In this paper, we fix the local transition function to $f_{150}$ for 1-dimensional 2-state 3 neighborhood cellular automata with a finite cyclic cell array. We discuss the reversibility of CA-150 with extended local structure and show a necessary and sufficient condition for CA-150 to be reversible.

\section{Preliminaries}

A cellular automaton $(Q, S, f, N)$ treated in the paper is a transition system $\left(Q^{S}, F\right)$ where $Q$ is the state set $\{0,1\}, m$ is a positive integer, $S=\mathbb{Z} / m \mathbb{Z}$ is the space of cells, $N=\left(n_{1}, n_{2}, \cdots, n_{s}\right)\left(n_{i} \in \mathbb{Z} / m \mathbb{Z}\right)$ is a neighborhood and $f$ is a local transition function from $Q^{s}$ into $Q$. The global transition function $F: Q^{S} \rightarrow Q^{S}$ is defined by $(f, N)$ as follows;

$$
F(x)(i)=f\left(x_{i+n_{1}}, x_{i+n_{2}}, \cdots, x_{i+n_{s}}\right)
$$

where the $j$ th cell $x_{j}$ of $x \in Q^{S}$ represents $x_{j \bmod m}$ for any integer $j$. The tuple $(f, N)$ is called a local structure of a cellular automaton $(Q, S, f, N)$. An element of $Q^{S}$ is called a configuration. A configuration is represented by a sequence of elements of $Q$. To easily express configurations, we write the sequence consisting of $n$ repetitions of $l$ as $l^{n}$ for a sequence $l$ of $Q$. For example, $0^{5}=00000$ and $(1011)^{3}=101110111011$.

In the paper we investigate the reversibility of cellular automata. A cellular automaton is called reversible if its global transition function is reversible and the global transition function is called reversible if it is injective. Since $S=\mathbb{Z} / m \mathbb{Z}$ is finite, then the injectivity and the surjectivity of the global transition function of a cellular automaton dealt with in the paper are equivalent.

Definition 2.1. The local structures $(f, N)$ and $\left(f^{\prime}, N^{\prime}\right)$ of cellular automata $(Q, S, f, N)$ and $\left(Q, S, f^{\prime}, N^{\prime}\right)$ are called equivalent if the global transition functions defined by $(f, N)$ and $\left(f^{\prime}, N^{\prime}\right)$ are equal, and it is represented in $(f, N) \approx\left(f^{\prime}, N^{\prime}\right)$ or $(Q, S, f, N)=\left(Q, S, f^{\prime}, N^{\prime}\right)$

Definition 2.2. Let $(X, F)$ and $\left(X^{\prime}, F^{\prime}\right)$ be two transition systems. An isomorphism $t:(X, F) \rightarrow\left(X^{\prime}, F^{\prime}\right)$ is a bijection $t: X \rightarrow X^{\prime}$ rendering the following square commutative:

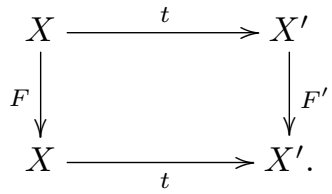

We call $(X, F)$ and $\left(X^{\prime}, F^{\prime}\right)$ isomorphic if there exists an isomorphism between $(X, F)$ and $\left(X^{\prime}, F^{\prime}\right)$. 
It is trivial that isomorphic dynamical systems are essentially the same ones.

Definition 2.3. For a local structure $(f, N)$ its symmetry local structure $\left(f^{\sharp}, N^{\sharp}\right)$ is defined as follows;

- $f^{\sharp}\left(q_{1}, q_{2}, \cdots, q_{s}\right)=f\left(q_{s}, q_{s-1}, \cdots q_{1}\right)$,

- $N^{\sharp}=\left(-n_{s},-n_{s-1}, \cdots,-n_{1}\right)$ for $N=\left(n_{1}, n_{2}, \cdots, n_{s}\right)$.

Definition 2.4. For a neighborhood $N=\left(n_{1}, n_{2}, \cdots, n_{s}\right), N^{\prime}=\left(n_{1}^{\prime}, n_{2}^{\prime}, \cdots, n_{s}^{\prime}\right)$ is called shift of $N$ if there exists some integer $\alpha \in \mathbb{Z}$ such that $n_{i}^{\prime}=n_{i}+\alpha$ for any $i$ $(1 \leq i \leq s)$.

Definition 2.5. Let $N=\left(n_{1}, n_{2}, \cdots, n_{s}\right)$ be a neighborhood and $\pi$ a permutation on $\{1,2, \cdots, s\}$. Then for $N$ and a local transition function $f: Q^{s} \rightarrow Q$ we define $N^{\pi}$ and $f^{\pi}: Q^{s} \rightarrow Q$ as follows;

- $N^{\pi}=\left(n_{\pi(1)}, n_{\pi(2)}, \cdots, n_{\pi(s)}\right)$,

- $f^{\pi}\left(x^{\pi}\right)=f(x)$.

where $x^{\pi}(i)=x(\pi(i))$ for $x \in Q^{s}$.

The following properties hold for the reversibility of cellular automata with any local function $f$ and any neighborhood $N$.

LEMma 2.6. For a cellular automaton $(Q, S, f, N)$ the following hold;

1. $(Q, S, f, N)$ and $\left(Q, S, f^{\sharp}, N^{\sharp}\right)$ are isomorphic.

2. If $N^{\prime}$ is shift of $N$ then the reversibilities of $(Q, S, f, N)$ and $\left(Q, S, f, N^{\prime}\right)$ are equivalent.

3. For any permutation $\pi$ for $N,(Q, S, f, N)$ and $\left(Q, S, f^{\pi}, N^{\pi}\right)$ are isomorphic.

For any configurations $x, x^{\prime} \in Q^{m}$ we define a addition of $x$ and $x^{\prime}$ as follows;

$$
\left(x+x^{\prime}\right)(i)=x(i)+x^{\prime}(i)(\bmod 2)
$$

A global transition function $F: Q^{m} \longrightarrow Q^{m}$ is called additive if the equation $F\left(x+x^{\prime}\right)=$ $F(x)+F\left(x^{\prime}\right)$ holds for any $x, x^{\prime} \in Q^{m}$, and a cellular automaton is called additive if its global transition function is additive. For the reversibility of additive cellular automata the following lemma is shown by Inokuchi, Honda et al. (2005).

Lemma 2.7. An additive global transition function $F: Q^{m} \longrightarrow Q^{m}$ is reversible if and only if $F(x)=0^{m}$ implies $x=0^{m}$ for all configuration $x \in Q^{m}$. 


\section{Reversibility of CA-150 with symmetry local structure}

Generally CA-150 is one of elementary cellular automata with $Q=\{0,1\}$ and $N=(-1,0,1)$. CA-150 have the local function $f_{150}: Q^{3} \longrightarrow Q$.

$$
f_{150}(a, b, c)=a+b+c \quad(\bmod 2) \quad(a, b, c \in Q)
$$

In this section we discuss the reversibility of the cellular automaton CA-150 with a symmetric neighborhood $N=(-t, 0, t)$ for a positive integer $t$.

For any neighborhood $N$ the cellular automaton $\left(Q, S, f_{150}, N\right)$ is additive. And since $f_{150}^{\sharp}=f_{150}$ and $f_{150}^{\pi}=f_{150}$ for any permutation $\pi$ for $N$, the following hold by lemma 2.6.

- The reversibilities of $\left(Q, S, f_{150}, N\right)$ and $\left(Q, S, f_{150}, N^{\sharp}\right)$ are equivalent.

- If $N^{\prime}$ is shift of $N$ then the reversibilities of $\left(Q, S, f_{150}, N\right)$ and $\left(Q, S, f_{150}, N^{\prime}\right)$ are equivalent.

- For any permutation $\pi$ for $N$ the reversibilities of $\left(Q, S, f_{150}, N\right)$ and $\left(Q, S, f_{150}, N^{\pi}\right)$ are equivalent.

In order to prove Theorem 3.3 we will show the following two lemmas.

Lemma 3.1. For the cellular automaton $\left(Q, \mathbb{Z} / m \mathbb{Z}, f_{150},(-t, 0, t)\right)$ of $m \in \mathbb{Z}$ and $x \in Q^{m}$, if $F(x)=0^{m}$ then the equation $x_{i}=x_{i+3 t}$ holds.

Proof. Assume that $F(x)=0^{m}$ for $x \in Q^{m}$. That is, $F(x)(i)=x_{i-t}+x_{i}+x_{i+t}=$ 0 for any $i \in \mathbb{Z} / m \mathbb{Z}$. Then

$$
\begin{aligned}
0 & =F(x)(i+t)+F(x)(i+2 t) \\
& =x_{i}+x_{i+t}+x_{i+2 t}+x_{i+t}+x_{i+2 t}+x_{i+3 t} \\
& =x_{i}+x_{i+3 t} .
\end{aligned}
$$

Therefore we have $x_{i}=x_{i+3 t}$.

LEMma 3.2. Let $m, m^{\prime}$ and $t$ be positive integers. If $m^{\prime}=m(\bmod 3 t)$ and the cellular automaton $\left(Q, \mathbb{Z} / m \mathbb{Z}, f_{150},(-t, 0, t)\right)$ is reversible, then the cellular automaton $\left(Q, \mathbb{Z} / m^{\prime} \mathbb{Z}, f_{150},(-t, 0, t)\right)$ is also reversible.

Proof. Let $F$ and $F^{\prime}$ be the global transition functions of $\left(Q, \mathbb{Z} / m \mathbb{Z}, f_{150},(-t, 0, t)\right)$ and $\left(Q, \mathbb{Z} / m^{\prime} \mathbb{Z}, f_{150},(-t, 0, t)\right)$ respectively, and assume that $F^{\prime}\left(x^{\prime}\right)=0^{m^{\prime}}$ for a configuration $x^{\prime} \in Q^{m^{\prime}}$. The equation $x_{i}^{\prime}=x_{i+3 t}^{\prime}$ holds for any integer $i$ from Lemma 3.1 and we can write $m^{\prime}=3 t \times n+m$ for some integer $n$ from $m^{\prime}=m(\bmod 3 t)$. Now we let $G C D\left(m^{\prime}, 3 t\right)=G C D(m, 3 t)=\alpha$. Then $x^{\prime}$ should be $\left(x_{1}^{\prime} x_{2}^{\prime} \cdots x_{\alpha}^{\prime}\right)^{\frac{m^{\prime}}{\alpha}}$. And set $x=\left(x_{1}^{\prime} x_{2}^{\prime} \cdots x_{\alpha}^{\prime}\right)^{\frac{m}{\alpha}} \in Q^{m}$ and we have $F(x)=0^{m}$ by the assumption $F^{\prime}\left(x^{\prime}\right)=0^{m^{\prime}}$. Since $\left(Q, \mathbb{Z} / m \mathbb{Z}, f_{150}, N\right)$ is reversible we have $x=$ $\left(x_{1}^{\prime} x_{2}^{\prime} \cdots x_{\alpha}^{\prime}\right)^{\frac{m}{\alpha}}=0^{m}$. Therefore $x^{\prime}=\left(x_{1}^{\prime} x_{2}^{\prime} \cdots x_{\alpha}^{\prime}\right)^{\frac{m^{\prime}}{\alpha}}=0^{m}$, that is, $\left(Q, \mathbb{Z} / m^{\prime} \mathbb{Z}, f_{150},(-t, 0, t)\right)$ is reversible. 
Inokuchi, Honda et al. (2005) discussed the reversibility of the 1-dimensional 2-states 3neighborhood cellular automata and showed conditions of the cell size $m$ for the cellular automata to be reversible. In the next theorem a relationship between $m$ and the neighborhood radius $t$ for the extended neighborhood version of $\left(Q, \mathbb{Z} / m \mathbb{Z}, f_{150},(-1,0,1)\right)$ to be reversible is given.

TheOREM 3.3. For any positive integers $t$ and $m$, the cellular automaton $\left(Q, \mathbb{Z} / m \mathbb{Z}, f_{150},(-t, 0, t)\right)$ is reversible if and only if $G C D(3 t, m)=G C D(t, m)$.

Proof. Let $F$ be the global transition function of $\left(Q, \mathbb{Z} / m \mathbb{Z}, f_{150},(-t, 0, t)\right)$. Assume that $F(x)=0^{m}$ for $x \in Q^{m}$.

1. In the case of $G C D(3 t, m)=1$.

For $\forall i \in \mathbb{N}$ and any integers $a, b$ the equation $x_{i}=x_{i+a \times 3 t+b \times m}$ holds by lemma 3.1. Since the indeterminate equation $a \times 3 t+b \times m=1$ has an integer solution by $G C D(3 t, m)=1$, we have $x_{i}=x_{i+a \times 3 t+b \times m}=x_{i+1}$. Therefore $x_{1}=x_{2}=\cdots=$ $x_{m}$, that is , $x=1^{m}$ or $x=0^{m}$. If $x=0^{m}$ then $F(x)=0^{m}$. But if $x=1^{m}$ then $F(x)=1^{m}$ and it contradicts the assumption. Hence we have $x=0^{m}$ and also that the cellular automaton $\left(Q, \mathbb{Z} / m \mathbb{Z}, f_{150},(-t, 0, t)\right)$ is reversible by lemma 2.7 .

2. In the case of $G C D(3 t, m)=m$ and $G C D(t, m)=m$.

Let $t=h m$ for some integer $h$. Then $x_{i+t}=x_{i+h m}=x_{i}$ and $x_{i-t}=x_{i-h m}=x_{i}$. Therefore

$$
0=F(x)(i)=x_{i-t}+x_{i}+x_{i+t}=3 x_{i}
$$

and we have $x_{i}=0$. Hence $x=0^{m}$ and the cellular automaton is reversible.

3. In the case of $G C D(3 t, m)=m$ and $G C D(t, m) \neq m$.

Since $m$ is a multiple of 3 we can write $m=3 m^{\prime}$ for some integer $m^{\prime}$ and $G C D\left(t, m^{\prime}\right)=m^{\prime}$ If $t$ is any multiple of 3 then $t$ should be a multiple of $m$. This contradicts the assumption, that is, $t$ is not any multiple of 3 . Then we can write $t=l m^{\prime}$ for some integer $l$ such that $l \neq 0(\bmod 3)$ because of $G C D\left(t, m^{\prime}\right)=m^{\prime}$. We have

$$
\begin{aligned}
0 & =F(x)(i) \\
& =x_{i-t}+x_{i}+x_{i+t} \\
& =x_{i-t+a m}+x_{i}+x_{i+t+b m} \\
& =x_{i+(3 a-l) m^{\prime}}+x_{i}+x_{i+(3 b+l) m^{\prime}} \\
& =x_{i}+x_{i+m^{\prime}}+x_{i+2 m^{\prime}}
\end{aligned}
$$

for any integers $a$ and $b$. Therefore $F\left(1^{m^{\prime}} 1^{m^{\prime}} 0^{m^{\prime}}\right)=0^{m}$, that is, $F$ is not reversible.

4. In the case of $G C D(3 t, m)=\alpha(1<\alpha<m)$ and $G C D(t, m)=\alpha$.

Since $x_{i}=x_{i+3 t}$ and we can write $m=h \alpha$ and $3 t=h^{\prime} \alpha$ for some integers $h$ and $h^{\prime}$, we have $x_{i}=x_{i+m a+3 t b}=x_{i+\left(h a+h^{\prime} b\right) \alpha}$ for any integers $a$ and $b$. Then there exist integers $a$ and $b$ such that $h a+h^{\prime} b=1$ since $h$ and $h^{\prime}$ are relatively prime. Hence we have $x_{i}=x_{i+\alpha}$ and we can write $x=\left(x_{1} x_{2} \cdots x_{\alpha}\right)^{\frac{m}{\alpha}}$. Let $m=l \alpha$ and 
$t=l^{\prime} \alpha$ for some integer $l$ and $l^{\prime}$. Then we have

$$
\begin{aligned}
0 & =F(x)(i) \\
& =x_{i-t}+x_{i}+x_{i+t} \\
& =x_{i-l^{\prime} \alpha}+x_{i}+x_{i+l^{\prime} \alpha} \\
& =3 x_{i} .
\end{aligned}
$$

Hence we have $x_{i}=0$ for any $i$, that is, $F$ is reversible.

5. In the case of $G C D(3 t, m)=\alpha(1<\alpha<m)$ and $G C D(t, m) \neq \alpha$.

We have $x_{i}=x_{i+\alpha}$ by the same discussion as above. And we can write $3 t=h \alpha$ and $m=h^{\prime} \alpha$ for some integers $h$ and $h^{\prime}$. Since $G C D(t, m)=G C D\left(\frac{h}{3} \alpha, h^{\prime} \alpha\right) \neq \alpha$, $h$ is not any multiple of 3 . And $\alpha$ should be a multiple of 3 since $t$ is a integer. Hence we have $G C D(t, m)=G C D\left(h \frac{\alpha}{3}, 3 h^{\prime} \frac{\alpha}{3}\right)=\frac{\alpha}{3}$ and

$$
\begin{aligned}
0 & =F(x)(i) \\
& =x_{i-t}+x_{i}+x_{i+t} \\
& =x_{i-h \times \frac{\alpha}{3}}+x_{i}+x_{i+h \times \frac{\alpha}{3}} \\
& =x_{i-h \times \frac{\alpha}{3}+a \alpha}+x_{i}+x_{i+h \times \frac{\alpha}{3}+b \alpha} \\
& =x_{i+(3 a-h) \times \frac{\alpha}{3}}+x_{i}+x_{i+(3 b+h) \times \frac{\alpha}{3}} \\
& =x_{i}+x_{i+\frac{\alpha}{3}}+x_{i+2 \frac{\alpha}{3}} .
\end{aligned}
$$

for any integers $a$ and $b$. Therefore $F\left(\left(1^{\frac{\alpha}{3}} 1^{\frac{\alpha}{3}} 0^{\frac{\alpha}{3}}\right)^{\frac{3 m}{\alpha}}\right)=0^{m}$, that is, $F$ is not reversible.

\section{Conclusion}

In this paper the relationship between the neighborhood radius and the cell size was clarified for reversible CA-150 when the neighborhood is extended symmetrically. If the neighborhood is restricted to symmetric then it can be easily determined whether CA-150 is reversible or not. Many people have analyzed the reversibility of cellular automata with a neighborhood consisting nearest cells, but few reports have been made on the behaviors of cellular automata with an changed local structure.

One of future works is to discuss the relationship between extended neighborhood and cell sizes for other additive cellular automata to be reversible. And also the authors will investigate the reversibility of non additive cellular automata with a changed local structure.

\section{Acknowledgement}

The authors thank the anonymous reviewers for their valuable comments.

\section{References}

Bhattacharjee, K. and Das, S. (2016). Reversibility of d-state finite cellular automata, Journal of Cellular Automata, 11(2-3), 213-245. 
Bhattacharjee, K. and Das, S. (2019). On Finite 1-Dimensional Cellular Automata: Reversibility and Semi-reversibility, arXiv preprint arXiv:1903.06011.

Feynman, R. (1982). Simulation Physics with Computers, International Journal of Theoretical Physics Vol. 21, No. 6\&7, pp. 467-488. 1982.

Inokuchi, S., Honda, K., Lee, H. Y., Sato, T., Mizoguchi, Y. and Kawahara, Y. (2005). On Reversible Cellular Automata with Finite Cell Array, Proceedings of Fourth International Conference on Unconventional Computation, LNCS 3699.

Inokuchi, S. and Mizoguchi, Y. (2005). Generalized Partitioned Quantum Cellular Automata and Quantization of Classical CA, Int. Journ. of Unconventional Computing, Vol. 1, 149-160.

Inokuchi, S., Mizoguchi, Y., Lee, H. Y. and Kawahara, Y. (2008). Periodic Behaviors of Quantum Cellular Automata, Bull. of Informatics and Cybernetics, Vol. 40, $17-50$.

Inui, N., Inokuchi, S., Mizoguchi, Y. and Konno, N. (2005). Statistical Properties of A Quantum Cellular Automaton, Physical Review A, Vol.72, 032323.

Morita, K. (1995). Reversible Simulation of One-dimensional Irreversible Cellular Automata, Theoretical Computer Science, Vol. 148 , 1, 157-163.

Morita, K. and Harao, M. (1989). Computation Universality of One-dimensional Reversible (Injective) Cellular Automata, Transactions of the IEICE, E 72, 758-762.

Nishio, H. (2006). How does the Neighborhood Affect the Global Behavior of Cellular Automata?, ACRI2006, LNCS 4173, 122-130.

Nishio, H. (2007). Changing the Neighborhood of Cellular Automata, MCU2007, LNCS 4664, 255-266.

Schumacher, B. and Werner, R. (2004). Reversible Quantum Cellular Automata, arXiv:quant-ph/0405174.

Watrous, J. (1995). On One-Dimensional Quantum Cellular Automata, Proceedings of the 36th Annual Symposium on Foundations of Computer Science, 528-537.

Wolfram, S. (2002). A New Kind of Science, Wolfram Media, Inc.

Received: October 3, 2021

Revised: November 13, 2021

Accept: November 15, 2021 